\title{
New Insights into Pseudo-Fractional ARMA Modelling
}

\author{
Manuel D. Ortigueira \\ UNINOVA and DEE ${ }^{1}$ \\ Faculdade de Ciências e Tecnologia da UNL \\ Campus da FCT da UNL, Quinta da Torre, \\ 2829 - 516 Caparica, Portugal \\ mdo@uninova.pt
}

\author{
António J. Serralheiro \\ Academia Militar and INESC ID \\ R. Alves Redol, 9, $2^{\circ}$, \\ 1000-029 Lisboa, Portugal \\ antonio.serralheiro@inesc-id.pt
}

\begin{abstract}
In this paper the modeling of Fractional Linear Systems through ARMA models is addressed. This study is performed by using a recursive algorithm for Impulse Response ARMA modelling leading us to propose suitable models for this problem
\end{abstract}

\section{INTRODUCTION}

ARMA models are suitable representations of processes with rational spectra and are widely used. It is useless to refer their importance in applications. There are a lot of algorithms to perform ARMA modelling in the stochastic case. In Impulse Response modelling the well known Padé algorithm is frequently used [1]. In this paper we shall not be concerned with the estimation task involved in ARMA modelling; instead, we are going to look at the underlying structure of the ARMA model in order to find alternative relations to obtain the ARMA parameters from the Impulse Response. To be more specific, consider the usual theoretical approach for computing the $\operatorname{ARMA}(\mathrm{N}, \mathrm{M})$ parameters from the Impulse Response, $h_{n}$ :

$$
\sum_{i=0}^{N} a_{i} h_{j-i}= \begin{cases}b_{j} & j \geq 0, \ldots, M \\ 0 & j>M\end{cases}
$$

where ai and bi are the AR and MA parameters. A close look into equation (1) shows that, to compute the ARMA $(N, M)$ parameters, we only need the first $\mathrm{N}+\mathrm{M}+1$ values of the Impulse Response $\{h(n), n=0, \ldots, N+M\}$. Here we propose a new description of the double Levinson recursion presented in [2]. The algorithm consists of the recursive solution of the system obtained from (1) with $j=$ $M$ to $j=N+M$ for the AR coefficients followed by the use of the first $\mathrm{M}+\mathrm{l}$ equations to obtain the MA parameters. This algorithm gives us the possibility of determining the orders of the systems when looking at the pattern formed by a sequence of coeficients. We applied this algorithm in (pseudo) fractional ARMA modeling and we observed that the pattern does not point clearly the orders, but give us some insights into minimum orders.

The recursive algorothm is presented in section II, where we present also two examples of its application. In section III we describe the results concerning fractional modelling. At last, we will present some conclusions.

\section{THE ALGORITHM}

The procedure we are going to describe is very similar to the one presented in [2], but, instead of using the matricidal formulation, we adopt a Schur-like description [3] since it is more direct and easier to implement. To begin with, we consider (1) and introduce a function $\mathbf{f}_{M}^{N}(j), j=0,1, \ldots$, given by:

$$
\mathbf{f}_{M}^{N}(j)=\sum_{i=0}^{N} a_{M}^{N}(i) h_{j+M-i}
$$

where we enhance the orders $\mathrm{N}$ and $\mathrm{M}$. According to (1) this function has gaps for $j=1,2, \ldots N$. For $N=0$, we thus have:

$$
f_{M}^{0}(j)=h_{j+M} j=0,1, \ldots
$$

The algorithm described in [2] uses an adjoint system [4]. Here, we introduce an adjoint function defined by:

$$
\mathbf{g}_{M}^{N}(j)=\sum_{i=0}^{N} \gamma_{M}^{N}(i) h_{N-j+M-i}
$$

with

$\mathrm{g}_{\mathrm{M}}^{0}(\mathrm{j})=\mathrm{h}_{-\mathrm{j}+\mathrm{M}} \mathrm{j=0}, \mathrm{l}, \ldots$

As it is clear, $g_{M}(j)$ has gaps for $j=1,2, \ldots, N$, too. The solution of (1) is recursively constructed for successive values of $N$ from $N=1$ to $N=N_{0}$, where $N_{0}$ is a positive integer. To do it, assume that we have constructed the $(\mathrm{N}$ 1)th order functions $f_{M}^{N-1}(j)$ and $g_{M}^{N-1}(j) j=0,1, \ldots$ We will construct the Nth order functions by the recursions:

$\underset{f_{M}}{N}(j)=f_{M}^{N-1}(j)+K_{M}^{N} g_{M}^{N-1}(N-j)$

and

$g_{M}^{N}(j)=g_{M}^{N-1}(j)+H_{M}^{N} f_{M}^{N-1}(N-j)$

where $\mathrm{K}_{M}^{\mathrm{N}}$ and $\mathrm{H}_{M}^{\mathrm{N}}$ are obtained by forcing both functions to have a gap at $\mathrm{j}=\mathrm{N}$. We obtain

$\mathrm{K}_{\mathrm{M}}^{\mathrm{N}}=-\frac{\mathrm{f}_{\mathrm{M}}^{\mathrm{N}-1}(\mathrm{~N})}{\mathrm{g}_{\mathrm{M}}^{\mathrm{N}-1}(0)}$
$\mathrm{H}_{\mathrm{M}}^{\mathrm{N}}=-\frac{\mathrm{g}_{\mathrm{M}}^{\mathrm{N}-1}(\mathrm{~N})}{\mathrm{f}_{\mathrm{M}}^{\mathrm{N}-1}(0)}$

As it is easy to verify, we have also:

$$
\mathrm{g}_{\mathrm{M}}^{\mathrm{N}}(0)=\mathrm{f}_{\mathrm{M}}^{\mathrm{N}-1}(0)\left(1-\mathrm{K}_{\mathrm{M}}^{\mathrm{N}} \cdot \mathrm{H}_{\mathrm{M}}^{\mathrm{N}}\right)
$$

\footnotetext{
${ }^{1}$ Also with INESC ID
} 
If the system with Impulse Response $h_{n}$ is really an $\operatorname{ARMA}\left(\mathrm{N}_{0}, \mathrm{M}_{0}\right)$, we will have

$\mathrm{b}_{\mathrm{j}}=\mathrm{f}_{\mathrm{M}_{0}}^{\mathrm{N}}\left(\mathrm{j} \cdot \mathrm{M}_{0}\right) \quad \mathrm{j}=0, \ldots, \mathrm{M}_{0}$

For the AR coefficients we use the $K_{M}^{N}$ and $H_{M}^{N}$ sequences to obtain the so-called Double Levinson recursion [2]:

$a_{M}^{N}(i)=a_{M}^{N-1}(i)+K_{M}^{N} \gamma_{M}^{N-1}(N-i)$

and

$\gamma_{M}^{N}(i)=\gamma_{M}^{N-1}(i)+H_{M}^{N}{ }^{a_{M}^{N}-1}(N-i)$

with $\mathrm{i}=0,1, \ldots, \mathrm{N} \cdot \mathrm{K}_{\mathrm{M}}^{\mathrm{N}}$ and $\mathrm{H}_{\mathrm{M}}^{\mathrm{N}}$ are Generalized Reflection Coefficients. The Double Levinson recursion supplies us with a very important result, which will be useful in determining the orders of the model. This result is stated in the following theorem:

Theorem - Let $M \geq 0$ be an integer constant and $\mathrm{A}_{\mathrm{M}^{\prime}}^{\mathrm{N}}(\mathrm{z})$ and $\Gamma_{\mathrm{M}+1}^{\mathrm{N}}(\mathrm{z})$ the $\mathrm{Z}$ Transforms of $\mathrm{a}_{M^{(i)}}^{\mathrm{N}}$ and ${ }_{\gamma_{M+1}^{N}}^{N}$. The Nth degree polynomials $A_{M}^{N}(z)$ and $\Gamma_{M+1}^{N}$ (z) corresponding to $M$ and $M+1$ zeros, respectively, are, up to a constant, reverse polynomials:

$\Gamma_{\mathrm{M}+1}^{\mathrm{N}}(\mathrm{z})=\phi \cdot \mathrm{z}^{-\mathrm{N}} \cdot \mathrm{A}_{\mathrm{M}^{\left(\mathrm{z}^{-1}\right)}}^{\mathrm{N}}$

$\phi$ being the last coefficient of $\Gamma_{M+1}^{N}(z)$. For proof, see [2]. As consequence of this theorem we have:

$\mathrm{H}_{\mathrm{M}+\mathrm{l}}^{\mathrm{N}} \cdot \mathrm{K}_{\mathrm{M}}^{\mathrm{N}}=1$

Its proof is immediate from the theorem. This result is very interesting since it allows us to compute the correct orders: it is enough to nun the algorithm for $\mathrm{N}, \mathrm{M}$ values ranging from 0 to $N_{0}, M_{0}$ higher than the expected orders. For the correct $A R$ order and the correct plus one MA order the product is one. To exemplify we will consider two examples.

Example 1 - the AR case: consider an AR(3) with coefficients $\mathrm{a}=\left[\begin{array}{llll}1 & -1.0871 & 1.1961 & -0.4512\end{array}\right]$. In table 1 we show the $\mathrm{H}_{\mathrm{M}}^{\mathrm{N}} \cdot \mathrm{K}_{\mathrm{M}}^{\mathrm{N}}$ product pattern for $\mathrm{N}, \mathrm{M}=0,1,2,3,4$. In the table, "hv" means a high value obtained when the determinant of the underlying matrix is almost zero (small values of $f_{M}^{N}(0)$ after a product almost equal to one). This happens when the orders are oversized. From the table we conclude easily that the system is indeed an $\operatorname{AR}(3)$. It is interesting to remark that if we compute the poles and zero corresponding to an $\operatorname{ARMA}(4,1)$, the extra pole is cancelled by the zero.

\begin{tabular}{|l|l|l|l|l|}
\hline $\mathrm{NM}$ & $\mathbf{1}$ & 2 & 3 & 4 \\
\hline 1 & -0.012 & hv & 0.0082 & -2.5803 \\
\hline 2 & 0.6571 & hv & 1.1446 & 1.0401 \\
\hline 3 & 1 & hv & 1 & 1 \\
\hline 4 & hv & 6.6935 & 0.2955 & -2.1107 \\
\hline
\end{tabular}

Example 2 - the ARMA case: we consider now an $\operatorname{ARMA}(3,2)$ system defined by the previous $A R$ parameters and with $b=\left[\begin{array}{lll}1 & 1.2 & -1.6\end{array}\right]$ as MA parameters. In table 2 we show again the $\mathrm{H}_{\mathrm{M}}^{\mathrm{N}} \cdot \mathrm{K}_{\mathrm{M}}^{\mathrm{N}}$ product pattern for $\mathrm{N}, \mathrm{M}=0,1,2,3,4$. The table suggests the correct orders.

It is important to refer that:

a) Although the system is not minimum phase, we obtain the correct MA parameters.

b) If the MA order is the correct one but the AR one is oversized, there is no problem since the extra coefficients are zero.

The application of this algorithm to the pole/zero modelling of integer order continuous-time systems is also possible. To do it we only have to substitute above the Impulse Response by the sequence $\mathrm{hn}=$ $\frac{(-1)^{n}}{n !} m_{n}, n=0,1, \ldots$, where $m n$ is the sequence of the momenta of the Impulse Response of the continuous-time system given by: $m_{n}=\int_{0}^{\infty} h(t) t{ }^{n} d t$.

\begin{tabular}{|l|l|l|l|l|}
\hline NMM & 1 & 2 & 3 & 4 \\
\hline 1 & -0.0592 & 62.5179 & 0.0652 & -1.7805 \\
\hline 2 & 0.1984 & 0.9592 & 0.8598 & 0.9575 \\
\hline 3 & 0.0422 & -0.9930 & 1 & 1 \\
\hline 4 & 0.0165 & 0 & hv & 0.8159 \\
\hline
\end{tabular}

Table 2- $H_{M}^{N} \cdot K_{M}^{N}$ product pattem for the $\operatorname{ARMA}(3,2)$ case

The importance of (14) lies on the bridge it establishes between two different MA order polynomials. As consequence of this theorem we have, from (13) [5]:

$A_{M}^{N-1}(z)=A_{M-1}^{N}(z)+\mu_{M}^{N} z^{-1} \cdot A_{M-1}^{N-1}(z)$

where $\mu_{M}^{N}$ is obtaining by forcing the Nth order coefficient to be zero:

$\mu_{M}^{N}=-\frac{a_{M-1}^{N}(N)}{a_{M-1}^{N-1}(N-1)}$

The recursion (16) is very interesting since it allows us to compute the $A R$ part of a given $\operatorname{ARMA}\left(N_{0}, M_{0}\right)$ from a sequence of $A R$ models with orders ranging from 1 to $\mathrm{N}_{0}+$ $M_{0}$. Using (2) we obtain

$\mathrm{f}_{M}^{N-1}(j)=f_{M-1}^{N}(j+1)+\mu_{M}^{N} f_{M-1}^{N-1}(j)$

that allows us to compute the $M A$ parameters from $f_{0}^{N}(j)$, obtained from the Levinson recursion (12) with $M=0$. In our applications we preferred to compute the MA parameters from (1).

We applied the recursion (16) to the model used in example 1. Immediately at the first recursion $(M=1)$, all the polynomial with degree greater than or equal to 4 reproduced the AR polynomial we were looking for. The extra coefficients were zero. The same happened with the second at $M=2$. When we go behond the correct MA order the coefficient (17) becomes very high due to a division by a very small value (theoretically, zero). 


\section{APPLICATION TO PSEUDO-FRACTIONAL MODELLING}

In this section, we assess the estimation of ARMA models for approximating discrete-time fractional models. We considered the fractional difference

$\mathrm{H}_{\mathrm{bd}}(\mathrm{z})=\left(1-\mathrm{z}^{-1}\right)^{\alpha}, \quad|\mathrm{z}|>1$

and the Tustin (bilinear)

$H_{b i i}(z)=\left(\frac{1-z^{-1}}{1+z^{-1}}\right)^{\alpha} \quad|z|>1$

as approximations to the $\alpha$ order differintegrator. For each one, we applied the above described algorithms and tried to find patterns that pointed us towards minimum orders. The product $\mathrm{H}_{\mathrm{M}+1}^{\mathrm{N}} \cdot \mathrm{K}_{\mathrm{M}}^{\mathrm{N}}$ pattern does not tell much, but and as in the previous tables, we find that the lower diagonal values were smaller than those in the upper diagonal in both cases (19) and (20). This suggests that the MA order is more important than the AR one. In the following tables, we illustrate the behaviour of such patterns for the backwards difference and bilinear. We represented by a " 0 " the values less than 0.9 , by " 1 " the values between 0.9 and 1.1 and by " 2 " the values above 1.1 .

\begin{tabular}{|l|llllllllll|}
\hline $\mathrm{N} / \mathrm{M}$ & 1 & 2 & 3 & 4 & 5 & 6 & 7 & 8 & 9 & 10 \\
\hline 1 & 0 & 2 & 2 & 2 & 1 & 1 & 1 & 1 & 1 & 1 \\
\hline 2 & 0 & 0 & 2 & 2 & 2 & 2 & 2 & 1 & 1 & $1 \mid$ \\
\hline 3 & 0 & 0 & 0 & 2 & 2 & 2 & 2 & 2 & 2 & 2 \\
\hline 4 & 0 & 0 & 0 & 0 & 2 & 2 & 2 & 2 & 2 & 2 \\
\hline 5 & 0 & 0 & 0 & 0 & 0 & 2 & 2 & 2 & 2 & 2 \\
\hline 6 & 0 & 0 & 0 & 0 & 0 & 0 & 2 & 2 & 2 & 2 \\
\hline 7 & 0 & 0 & 0 & 0 & 0 & 0 & 0 & 2 & 2 & 2 \\
\hline 8 & 0 & 0 & 0 & 0 & 0 & 0 & 0 & 0 & 2 & 2 \\
\hline 19 & 0 & 0 & 0 & 0 & 0 & 0 & 0 & 0 & 0 & 2 \\
\hline 10 & 0 & 0 & 0 & 0 & 0 & 0 & 0 & 0 & 0 & 0 \\
\hline
\end{tabular}

Table $3-H_{M}^{N} \cdot K_{M}^{N}$ product pattern for the ARMA $(10,10)$ corresponding to the backward difference case

\begin{tabular}{|l|llllllllll|}
\hline N/M & 1 & 2 & 3 & 4 & 5 & 6 & 7 & 8 & 9 & 10 \\
\hline 1 & 0 & 2 & 0 & 2 & 0 & 2 & 0 & 2 & 0 & 2 \\
\hline 2 & 0 & 0 & 2 & 0 & 2 & 0 & 2 & 0 & 2 & 1 \\
\hline 3 & 0 & 0 & 0 & 2 & 0 & 2 & 0 & 2 & 0 & 2 \\
\hline 4 & 0 & 0 & 0 & 0 & 2 & 0 & 2 & 0 & 2 & 0 \\
\hline 5 & 0 & 0 & 0 & 0 & 0 & 2 & 0 & 2 & 0 & 2 \\
\hline 6 & 0 & 0 & 0 & 0 & 0 & 0 & 2 & 0 & 2 & 0 \\
\hline 17 & 0 & 0 & 0 & 0 & 0 & 0 & 0 & 2 & 0 & 2 \\
\hline 8 & 0 & 0 & 0 & 0 & 0 & 0 & 0 & 0 & 2 & 0 \\
\hline 19 & 0 & 0 & 0 & 0 & 0 & 0 & 0 & 0 & 0 & 2 \\
\hline 10 & 0 & 0 & 0 & 0 & 0 & 0 & 0 & 0 & 0 & 0 \\
\hline
\end{tabular}

Table 3- ${ }_{M}^{N} \cdot K_{M}^{N}$ product pattern for the ARMA(10,10) corresponding to the bilinear case

To try to find other insights into the orders, we ran the recursive algorithm. This allowed us to conclude that a) Small values of the coefficient $\mu_{M}^{N}$ in (17) points to correct $\mathrm{N}$ and $\mathrm{M}$ orders

b) Very high values of $\mu_{M}^{N}$ mean that, at least the MA order is higher than the correct. In this situation we must decrease it. This situation corresponds to unstable model.

c) The observation of the $\mu_{M}^{N}$ pattern suggests an $\operatorname{ARMA}(\mathrm{N}, \mathrm{N})$ model.

d) In the difference case, the poles and zeros of the ARMA(N,N) model are always positive and interlaced.

e) In the bilinear case, the poles and zeros of the ARMA(N,N) model are symmetric.

f) Our simulations pointed out to values of $\mathrm{N}$ from 7 to 10. For most situations the approximation is good in both time and frequency.
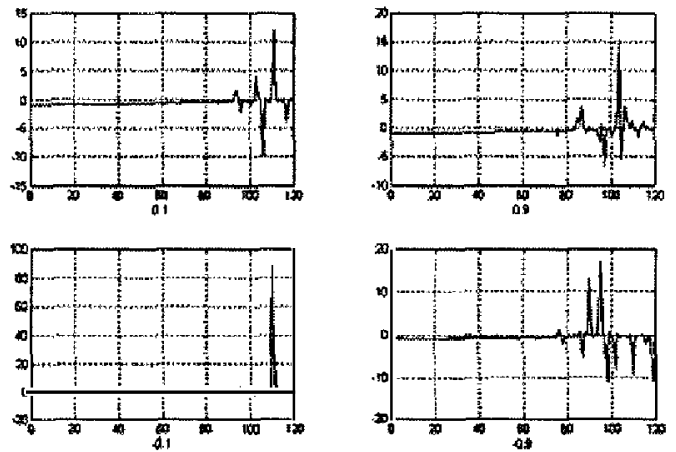

Figure I-the behaviour of the $\mu_{M}^{N}$ as a tool for orders choice, for the differentiator (above) and integrator (below), with $\alpha=.1$ (left) and $\alpha=0.9$ (right).
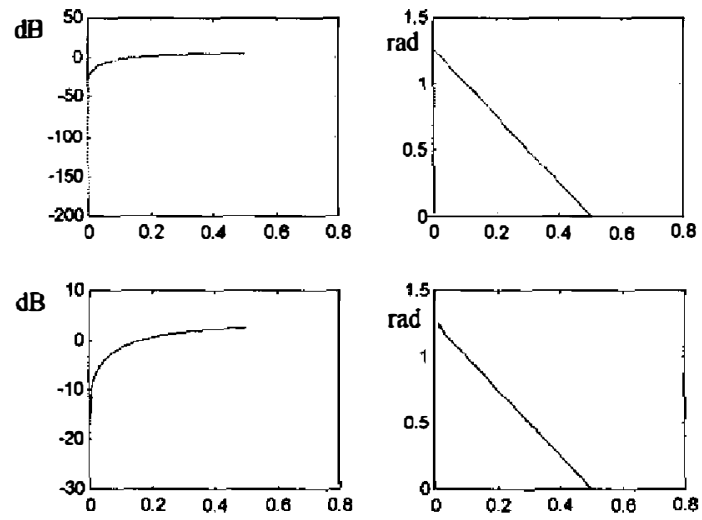

Figure $2-A R M A(N, M)$ frequency response plots (amplitude on the left and phase on the right hands) the for the backward difference with $\alpha=0.8$ for $N, M=1,3,5, \ldots, 15$. 
dB
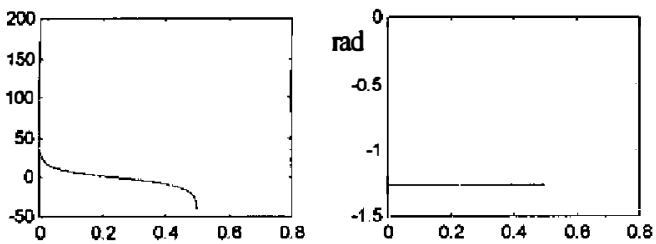

dB
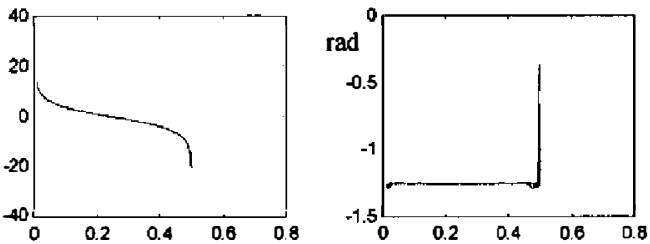

Figure $3-A R M A(N, M)$ frequency response plots (amplitude on the left and phase on the right hands) the for bilinear with $\alpha=$ 0.8 for $N, M=1,3,5, \ldots, 15$.

We made also a study of the pole-zero distribution (figures 4 and 5) and found that:

a) In the backward difference case the poles and zeros are positive real. With higher orders complex poles and zeros may appear.

b) In the bilinear case the poles and zeros are real. We did not find complex poles or zeros, even with high orders.

c) In the backward difference, we obtained unstable models for some higher orders.

d) If the pole order is the same of the zero order the poles and zeros are interlaced. In the bilinear case, they are symmetric each other.

e) If the orders are not equal, the "extra" poles or zeros tend to appear near zero.

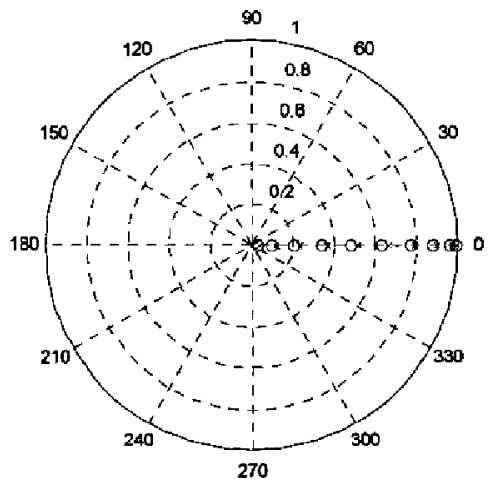

Figure 4 - ARMA(N,M) pole-zero plots the for backward differences with $\alpha=0.8$ for $N, M=10$.

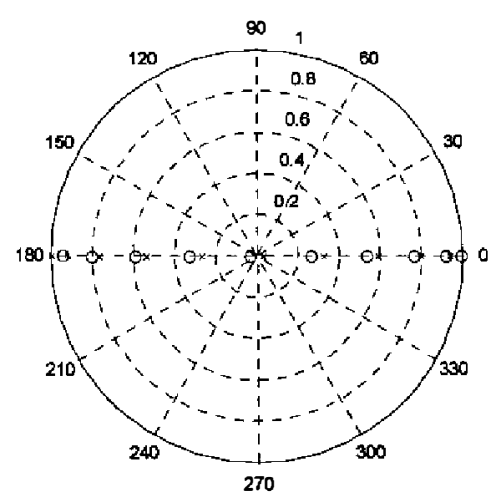

Figure 5-ARMA $(N, M)$ pole-zero plots the for bilinear with $\alpha=$ 0.8 for $N, M=10$.

\section{CONCLUSIONS}

The algorithm we have just presented gives a simple way of computing the $\mathrm{AR}$ and $\mathrm{MA}$ parameters of an ARMA model when the Impulse Response is known. The algorithm is recursive and gives insights into the orders computation. The application to the fracional modelling case suggested that we should use ARMA(N,N) models.

\section{REFERENCES}

[1] K. Kumar, "On the Identification of Autoregressive Moving Average Models", Joumal of Control and Intelligent Systems, Vol. 28, No2, pp 41-46, 2000.

[2] M. D. Ortigueira and J. M. Tribolet, "On the Double Levinson Recursion Formulation of ARMA Spectral Estimation," Proc. of IEEE ICASSP, 1983.

[3] E. A. Robinson and S. Treitel, " Maximum Entropy and the Relationship of the Partial Autocorrelation to the Reflection Coefficients of a Layered System, " IEEE Trans. on ASSP, Vol. 28, No. 2, April 1980.

[4] G. Carayannis, N. Kaloupsidis, and D. G. Manolakis, "Fast Algorithms for a Class of Linear Equations," IEEE Trans. on ASSP, Vol.30, No.2, pp. 227-239, 1982.

[5] Ortigueira, M.D., "ARMA Realization from the Reflection Coefficient Sequence", Signal Processing, vol. 32 No. 3, June 1993. 